occur, but with a systematic discrepancy between the strength of the stress components anticipated in the various directions of space and that actually found in quantitative measurements. The dis. orepancy was rectified, and full agreement reached with all experimental results obtained so far, in a revised theory, in which the arbitrarily assumed law of elasticity was replaced by an empirical law of the form

$$
\begin{aligned}
& \frac{P_{a u}-P_{b v}}{R_{a u}-\bar{R}_{b v}}=\frac{P_{b v}-P_{c w}}{R_{b v}-R_{c w}}= \\
& \frac{P_{c w}-P_{a u}}{R_{c w}-R_{a u}}=G(P, R)
\end{aligned}
$$

Here-for the energized state considered-we denote by $P_{k l}$ the $k l$ component of stress, by $R_{m n}$ the $m n$ component of the recoverable strain, measured on the classical scale as the elongation ratio squared minus unity, by $a, b, c$ and $u, v, w$ the numbers $1,2,3$ or any cyclical permutation of these numbers, and by $G(P, R)$ a function of the three fundamental scalar invariants of stress and strain, and other scalar parameters such as temperature, time, etc. The function $G(P, R)$ may be regarded as a generalized modulus of elasticity, the value of which may vary with the material and the rheological state, the temperature, time, etc. This is best seen when the empirical law (1) is specialized for the case of a stationary laminar shearing movement, where the suffixes 1, 2 and 3 correspond respectively to the directions of the lines of flow, the perpendicular to the shearing plane, and the normal to both these directions, and where the recoverable strain has components $R_{m n}$ which are all zero, except $R_{11}, R_{12}$, and $R_{81}$, with $R_{11}=\left(R_{12}\right)^{2}=\left(R_{21}\right)^{2}$.

Recently, further progress was made ${ }^{5}$ when we succeeded in replacing the empirical law of elasticity (1) by a simple theoretical assumption. We consider an elastic continuum in any energized state, and imagine it cut into two parts by a plane drawn in any direction through some arbitrarily selected point. For every such plane the traction force will define a triad of mutually perpendicular directions, according to its normal, tangential and crosscomponents; and a second triad is defined by taking similar components of the displacement of the two parts relative to one another when the continuum recovers to a ground-state. We can formally deduce equation (1) from the assumption of a coincidence of these two triads of directions for every plane. This assumption, and the elasticity law derived from it, have been confirmed empirically over wide ranges of materials and mechanical actions; but further experimental investigations are required, and are in fact under way, to ascertain the exact limitations of validity, since a theoretical proof can be given only under certain conditions. Keeping within the range of elastic continua which are isotropic in all the ground-states, we can prove the validity of the assumption $(a)$ in all classes of rheological states for certain orientations of planes, and $(b)$ for all possible orientations of planes in certain classes of rheological states, including all classes restricted either to infinitesimal small strains, or to any stresses and strains with cylindrical symmetry.

1 Lander, C. H., Melchett Lecture 1945, Institute of Fuel.

' Russell, R. J., Ph.D. Thesis, Imperial College, London, 1945.

- Garner, F. H., and Nissan, A. H., Nature, 158, 634 (1946).

' Weissenberg, K., Arch. Sci. Phys. et Nat., v, 17, 1 (1934). 'Welssenberg, K., Conference of the British Rheologists' Club,

\section{EMERGENCY SCIENTIFIC RESEARCH BUREAU, EIRE}

$T$ HE report of the Emergency Scientific Research Bureau, Eire, covering the period February 21, 1941-March $31,1945^{*}$, which was set up with Prof. J. J. Dowling as chairman, gives brief details of the work of the Bureau.

A large part of the Bureau's experimental work dealt with fuel problems, and the most important of these included the manufacture of charcoal from turf, the efficiency of mobile producer plants, and the production of town gas under emergency conditions. Work on the production of turf charcoal led to the design, erection and operation by the Turf Development Board, Ltd., of a plant on the Turraun Bog, from which charcoal suitable for use as a producer fuel was obtained with an output of up to 22 tons a week. Work on town gas resulted in a study of the possibilities of using mixtures of turf and coal, and in modifications in plant which to some extent eased the difficulties being experienced at one of the large gas works. One of the first problems studied was that of mobile gas producers, including the use of an electric filter for the gas; but although such a filter can be constructed, it is not much more efficient, is more expensive and requires much more careful servicing. The drying of turf and the development of a gas specific gravity meter were other investigations in the same field.

Much attention was given to chemicals for the manufacture of matches, including the manufacture both of phosphorus and of potassium chlorate; while a process was also worked out for the manufacture of sulphur by dry distillation from spent oxide. Plant was also developed for the supply of compressed carbon dioxide and ammonia for refrigeration purposes, for aluminium sulphate for water purification and paper manufacture, and for the manufacture of formalin by oxidation of methyl alcohol.

A geophysical survey of mineral deposits of economic importance, and the increase in output to $20 \mathrm{~kW}$. of the existing $\mathbf{1} \cdot 5 \mathrm{~kW}$. short-wave Marconi transmitter at Moydrum were other tasks undertaken.

The maintenance of such essential medical supplies as anæsthetic ether, glycerol, insulin, and vitamins, and of supplies of yeast and flour for breadmaking, and the manufacture of citric acid by fermentation were other subjects investigated.

Experimental work was also carried out on the production of carbon black by combustion of used lubricating oil, the spectrographic analysis of steel, production of ferro-silicon, electro-deposition of ductile pure iron, carburizing steel for knife-blades, refractory bricks, carbon electrodes, replacements for storage batteries, the recovery of zinc from used primary cells and the alkali treatment of Clare phosphate to give a satisfactory fertilizer. A process was developed for a photo-engraving glue from fish-skins. Work was done on acetone, and processes for ethyl acetate from 80 per cent acetic acid, for agar-agar from seaweed, for an insecticide by extraction of offal tobacco, and a substitute for cutch as a preservative for fishing nets, were also worked out by the Bureau. A modified method for the determination of fat in milk was devised. Advice was given on the use of substitutes for imported wool oil, and for economy and efficiency in the use of lubricating oils.

* Emergency Scientiflc Research Bureau. Report. Pp. 96. (Dublin : * Emergency Scientific Resear
Stationery Office, 1946.) 28. 